\title{
MMSE Joint Detection in frequency-selective wireless communication channels for DS-CDMA systems
}

\author{
Daniel P. Palomar, Javier R. Fonollosa and Miguel A. Lagunas ${ }^{1}$ \\ Universitat Politècnica de Catalunya \\ Department of Signal Theory and Communications \\ c/ Jordi Girona, 1-3, Mòdul D5, Campus Nord UPC \\ 08034 Barcelona (SPAIN) \\ e-mail: danielegps.tsc.upc.es
}

\begin{abstract}
In digital mobile communication systems, two important problems arise, namely multiple access interference (MAI) and intersymbol interference (ISI). An attractive solution is the use of code division multiple access (CDMA) systems possibly combined with TDMA and FDMA. In CDMA communication systems, the MAI and ISI can be efficiently combated by applying multi-sensor linear joint detection (JD) techniques, in particular, space-time processing methods. In most cases, they are derived from a symbol-level error criterion. However, in some other cases they are obtained from a chip-level viewpoint, also known as chip sequence restoral techniques. In the present paper, a detailed analytical comparison between chip-rate and symbol-rate minimum mean square error (MMSE) block filtering is provided, taking into account also other features such as structured vs. non-structured block filter computation and chiplevel vs. symbol-level training sequence. Monte-Carlo simulation results are also given to provide realistic comparisons of the different methods.
\end{abstract}

\section{INTRODUCTION}

In digital mobile communication systems, two major problems arise naturally, namely the multiple access interference (MAI), caused by the simultaneous transmission of signals associated with several active users that share the same transmission medium, and the intersymbol interference (ISI), arising from frequency-selective mobile radio channels that are, in general, time-varying [1]. An attractive solution to the multiple access problem is code division multiple access (CDMA) [2], possibly combined with FDMA and TDMA to yield a hybrid MA scheme, which is currently being standardized for the application to the third generation digital mobile radio systems, e.g. TDD ${ }^{2}$ mode of $\mathrm{UTRA}^{3}$ envisaged by the $3 \mathrm{GPP}^{1}$ [3].

In a CDMA mobile radio system, the MAI can be efficiently combated by applying either joint detection (JD) $[4,5,6]$ or interference cancellation (IC) techniques $[7,8]$. Furthermore,

\footnotetext{
${ }^{1}$ This work was partially supported by the European Comission under Project IST-1999-11729 METRA; the Spanish Government (CYCIT) TIC98-0412, TIC98-0703, TIC99-0849; and the Catalan Government (CIRIT) 1998SGR 00081, 1999FI 00588.

${ }^{2}$ TDD: Time Division Duplex.

${ }^{3}$ UTRA: UMTS (Universal Mobile Telecommunication System) Terrestrial Radio Access.

${ }^{1} 3$ GPP: $3^{\text {rd }}$ Generation Partnership Project.
}

the existence of ISI in conjunction with MAI has to be considered to account for frequency-selective channels. Unfortunately, the maximum likelihood (ML) detection method [0] is computationally unaffordable. Therefore, suboptimum JD techniques have to be devised $[6,9,10]$. In addition, the use of diversity techniques is welcome to overcome the fading of the channel. In [11], antenna diversity is exploited by deriving six different linear JD methods for antenna diversity combining.

Most of the existing literature for linear JD schemes has focussed on the derivation of block linear equalizers so that, after space-time block filtering, an estimation of the transmitted symbols (expected to be free of ISI and MAI) is given. However, an alternative approach can be obtained from a chiplevel viewpoint, being the objective in this case to find a linear block equalizer that gives at its output the transmitted chip sequence instead of the transmitted symbol sequence $[12,13]$. In [14], numerical comparisons for the downlink single-antenna case can be found.

In the present paper, only the multi-sensor minimum mean square-error (MMSE) approach has been considered, because it clearly outperforms the decorrelating matched filtering (DMF) and the zero-forcing (ZF) approaches $[11,10]$. As it will be seen, the estimation of the block-equalizer matrix can be performed following either a non-structured or a structured approach. In both cases, a training sequence is used ${ }^{5}$, which can be defined either on a chip or a symbol level. Taking into account all the aforementioned possible choices that can be made within the MMSE general approach, many potential different versions arise. In the following, these variants are analytically derived and compared. Monte-Carlo simulations were also performed to give realistic comparisons of the different methods when applied to TDD mode of UTRA (see the technical specification given by the 3GPP [3]).

In Section II, the signal model at chip and symbol level is defined. Expressions for symbol and chip rate space-time MMSE block filters are derived in Section III. In Sections IV$\mathrm{V}$, asymptotic analytical comparisons and performance evaluation criteria are given. Section VI is devoted to Monte-Carlo simulation results. And, finally, in section VII, the final conclusions are drawn.

\section{MULTIUSER-MULTISENSOR SIGNAL MODEL}

Since the goal is to develop array joint detection methods, a multiuser-multisensor signal model has to be defined, i.e., the signals of the $K$ intracell users (transmitting with one antenna) received at each antenna, $1 \leq q \leq Q$, of the base station (BS) array are explicitly written and the rest (intercell

${ }^{5}$ It is also possible to design the filter in a blind manner, but this is not considered in this paper. 
users, other interfering signals and thermal noise) are lumped together in the noise vector $\mathbf{n}$ :

$$
\mathbf{x}_{q}=\sum_{k=1}^{K} \mathbf{H}_{c, q}^{(k)} \mathbf{s}_{c}^{(k)}+\mathbf{n}_{q}
$$

where $\mathbf{s}_{c}^{(k)} \in \mathbb{C}^{N_{c} x 1}$ is a column vector corresponding to the temporal chip sequence transmitted by user $k$ of length $\mathrm{N}_{c}$; $\mathbf{H}_{c, q}^{(k)} \in \mathbb{C}^{\left(\mathrm{N}_{c}+\mathrm{L}_{c}-1\right) \times \mathrm{N}_{c}}$ is the convolution matrix of the channel from user $k$ to antenna $q$ of length $\mathrm{L}_{c} ; \mathbf{n}_{q} \in \mathbb{C}^{\left(\mathrm{N}_{\mathrm{c}}+\mathrm{L}_{c}-1\right) \times 1}$ and $\mathbf{x}_{q} \in \mathbb{C}^{\left(\mathrm{N}_{c}+\mathrm{L}_{c}-1\right) \times 1}$ are the noise and the total received signals at antenna $k$ respectively. The signal model can be further compacted by stacking columnwise the signals of each antenna:

$$
\mathbf{x}=\sum_{k=1}^{K} \mathbf{H}_{c}^{(k)} \mathbf{s}_{c}^{(k)}+\mathbf{n}=\mathbf{H}_{c} \mathbf{s}_{c}+\mathbf{n}
$$

where $\mathbf{H}_{c}=\left[\begin{array}{lll}\mathbf{H}_{c}^{(1)} & \ldots & \mathbf{H}_{c}^{(K)}\end{array}\right]$,

$$
\begin{aligned}
& \mathbf{H}_{c}^{(k)}=\left[\begin{array}{llll}
\mathbf{H}_{c, 1}^{(k) T} & \ldots & \mathbf{H}_{c, Q}^{(k) T}
\end{array}\right]^{T}, \text { and } \\
& \mathbf{s}_{c}=\left[\begin{array}{lll}
\mathbf{s}_{c}^{(1) T} & \cdots & \left.\mathbf{s}_{c}^{(K) T}\right]^{T} .
\end{array}\right.
\end{aligned}
$$

Assuming that a short spreading code is being used, the transmitted chip sequence by user $k$ can be expressed as $\mathbf{s}_{c}^{(k)}=\mathbf{C}^{(k)} \mathbf{s}^{(k)}$, where $\mathbf{s}^{(k)} \in \mathbb{C}^{\mathrm{Nx} \mathbf{1}}$ corresponds to the transmitted symbol sequence; $\mathbf{C}^{(k)}=\mathbf{I}_{N} \otimes \mathbf{c}^{(k)}$, being $\mathbf{c}^{(k)} \in \mathbb{C}^{\mathrm{SF} \times 1}$ the normalized spreading code of user $k$ with a spreading factor of $\mathrm{SF}$ and $\mathrm{N}_{c}=\mathrm{N} \cdot \mathrm{SF}$. It can be compactly expressed on a multiuser fashion using stacked vectors as $\mathbf{s}_{c}=\mathbf{C s}$, where $\mathbf{C}=\operatorname{diag}\left\{\left[\begin{array}{lll}\mathbf{C}^{(1)} & \cdots & \mathbf{C}^{(K)}\end{array}\right]\right\}$ is a block-diagonal matrix verifying $\mathbf{C}^{H} \mathbf{C}=\mathbf{I}$. The received signal model can be then rewritten as:

$$
\mathbf{x}=\mathbf{H}_{c} \mathbf{C s}+\mathbf{n}=\mathbf{H}_{s} \mathbf{s}+\mathbf{n}
$$

where $\mathbf{H}_{s}=\mathbf{H}_{c} \mathbf{C}$ (for a detailed definition of the structure of $\mathbf{H}_{s}$ see [11]).

Equations (2) and (3) are referred to as chip-level and symbol-level signal model respectively, because they relate the received signal to either the transmitted chip or symbol sequence, being the symbol-level signal model the most commonly used (e.g. $[9,11,10])$. Although the following derivations are derived using the presented multiuser uplink model, they also hold for downlink and for a single-user model (including the intracell users in the noise vector).

\section{SYMBOL/CHIP-RATE SPACE-TIME MMSE BLOCK FILTERING}

A linear space-time block filtering approach to estimate the multiuser transmitted sequence is assumed. It will aim to deliver the desired signal as free of intersymbol interference (ISI) and multi-access interference (MAI) as possible. The block filtering scheme can be developed either at chip or symbol rate, depending on whether it gives out the estimated transmitted chip sequence or symbol sequence (see Figure 1). The chiprate linear block filtering has also been termed elsewhere $P N$ sequence regeneration [12] and chip sequence restoral [13]; it requires a posterior despreading stage to get the symbol estimates.

The design of the space-time processing matrix we consider herein relies on a training sequence that can be defined either on a symbol or chip basis. For the case of symbol-level training sequence, the covariance matrix of the chip sequence is $\mathbf{R}_{c}=\mathbf{C R}_{s} \mathbf{C}^{H}$ (singular matrix), where $\mathbf{R}_{c}=E\left\{\mathbf{s}_{c} \mathbf{s}_{c}^{H}\right\}$ and $\mathbf{R}_{s}=E\left\{\mathbf{s s}^{H}\right\}$. For the case of chip-level training sequence, however, this matrix is completely unstructured (it does not

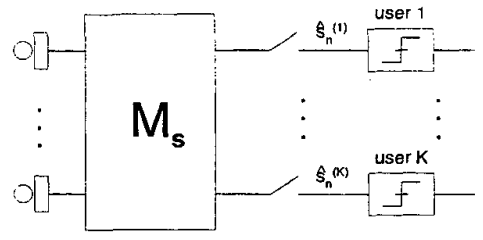

(a) Symbol-rate filtering

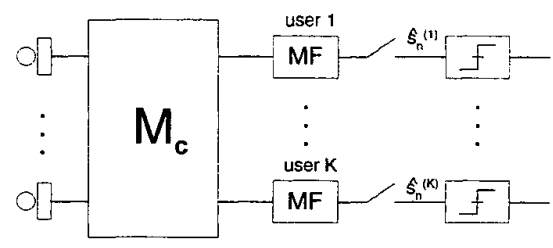

(b) Chip-rate filtering

Figure 1: Space-time filtering schemes.

contain the temporal structure of the codes but white-like PN sequences) $\mathbf{R}_{c}=\frac{1}{\mathrm{SF}} \mathbf{I}$ (full rank). It is important to remark that during the transmission of the data symbols, the covariance matrix of the chip-level transmitted sequence corresponds, indeed, to the structured expression, therefore, we can foresee that the use of the structured $\mathbf{R}_{c}$ in the filter design will yield a better performance.

\section{SYMBOL-RATE BLOCK FILTERING}

The symbol-rate MMSE space-time block filtering minimizes the mean square error (MSE) between its output and the desired sequence at a symbol level:

$$
\min E\left\{\|\mathbf{s}-\hat{s}\|^{2}\right\}
$$

where $\hat{\mathbf{s}}=\mathbf{M}_{s} \mathbf{x}$ (see Figure 1(a)) and matrix $\mathbf{M}_{s}$ is given by

$$
\begin{aligned}
\mathbf{M}_{s} & =E\left\{\mathbf{s x}^{H}\right\} E\left\{\mathbf{x x}^{H}\right\}^{-1} \\
& =\mathbf{R}_{s} \mathbf{H}_{s}^{H}\left(\mathbf{H}_{s} \mathbf{R}_{s} \mathbf{H}_{s}^{H}+\mathbf{R}_{n}\right)^{-1} \\
& =\left(\mathbf{H}_{s}^{H} \mathbf{R}_{n}^{-1} \mathbf{H}_{s}+\mathbf{R}_{s}^{-1}\right)^{-1} \mathbf{H}_{s}^{H} \mathbf{R}_{n}^{-1}
\end{aligned}
$$

where $\mathbf{R}_{n}=E\left\{\mathbf{n n}^{H}\right\}$. Equations (6) and (7) are related by the matrix inversion lemma.

The non-structured expression for $\mathbf{M}_{s}$ (5) can be directly implemented (Wiener filter) by approximating the ensemble average by a temporal one (this can be done only if a reducedsize $\mathbf{M}_{s}$ corresponding to a sliding window is used) whenever the training sequence is given at a symbol level. On the other hand, the utilization of the structured expressions (6) and (7) requires a previous estimation of $\mathbf{H}_{s}$ or $\mathbf{H}_{c}$, which can be achieved by means of a symbol/chip-level training sequence respectively. See Figure 2 for a scheme of the different MMSE filtering versions (note the dashed lines relating equivalent approaches).

\section{ChIP-RATE BLOCK FILTERING}

The chip-rate MMSE space-time block filtering minimizes the chip-level MSE:

$$
\min E\left\{\left\|\mathbf{s}_{c}-\hat{\mathbf{s}}_{c}\right\|^{2}\right\}
$$




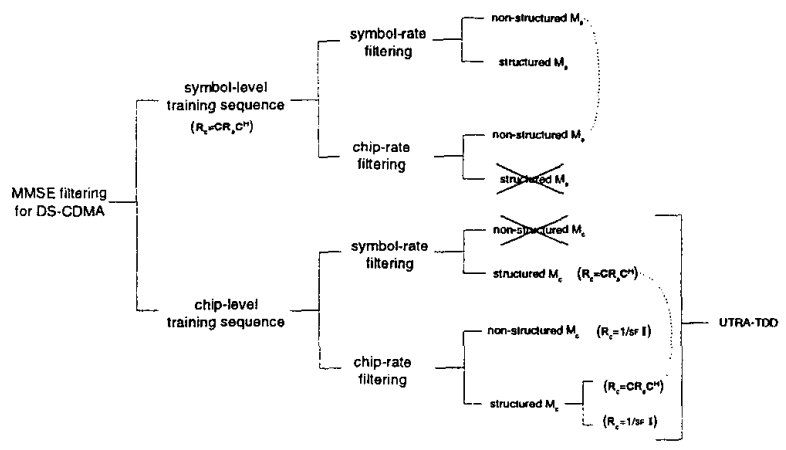

Figure 2: All possible time reference based-only MMSE spacetime filtering combination schemes.

where $\hat{\mathbf{s}}_{c}=\mathbf{M}_{c} \mathbf{x}$ and matrix $\mathbf{M}_{c}$ is given by:

$$
\begin{aligned}
\mathbf{M}_{c} & =E\left\{\mathbf{s}_{c} \mathbf{x}^{H}\right\} E\left\{\mathbf{x} \mathbf{x}^{H}\right\}^{-1} \\
& =\mathbf{R}_{c} \mathbf{H}_{c}^{H}\left(\mathbf{H}_{c} \mathbf{R}_{c} \mathbf{H}_{c}^{H}+\mathbf{R}_{n}\right)^{-1} .
\end{aligned}
$$

After the MMSE chip-rate filtering, a matched filtering or despreading stage is needed: $\hat{\mathbf{s}}=\mathbf{C}^{H} \hat{\mathbf{s}}_{c}$ (see Figure $1(\mathrm{~b})$ ).

Again, the non-structured approach (9) can be directly implemented by using a temporal average (Wiener filter). However, depending on whether the training sequence was generated on a symbol or chip basis it leads to different estimations. If symbol-level training sequence is used $\left(\mathbf{R}_{c}=\mathbf{C R}_{s} \mathbf{C}^{H}\right)$, then it is straightforward to check that the resulting combination of the chip-rate filtering and despreading stage is identical to the symbol-rate filtering:

$$
\mathbf{C}^{H} \mathbf{M}_{c}=\mathbf{R}_{s} \mathbf{H}_{s}^{H}\left(\mathbf{H}_{s} \mathbf{R}_{s} \mathbf{H}_{s}^{H}+\mathbf{R}_{n}\right)^{-1} .
$$

In case that chip-level training sequence is used $\left(\mathbf{R}_{c}=\frac{1}{\mathrm{SF}} \mathbf{I}\right)$, $\mathbf{R}_{c}$ is full rank and we can apply the matrix inversion lemma to obtain (as for the symbol-rate filtering case):

$$
\mathbf{C}^{H} \mathbf{M}_{c}=\mathbf{C}^{H}\left(\mathbf{H}_{c}^{H} \mathbf{R}_{n}^{-1} \mathbf{H}_{c}+\mathbf{R}_{c}^{-1}\right)^{-1} \mathbf{H}_{c}^{H} \mathbf{R}_{n}^{-1} .
$$

The structured approach requires a previous estimation of $\mathbf{H}_{c}$ and, therefore, a chip-level training sequence is needed. In any case, we can choose whether to use the structured or non-structured matrix $\mathbf{R}_{c}$. See Figure 2 for a scheme of all filtering possibilities.

\section{ASYMPTOTIC ANALYTICAL COMPARISON OF SPACE-TIME MMSE BLOCK FILTERING}

To compare analytically the performance of space-time MMSE block filtering schemes using both the structured and unstructured matrices $\mathbf{R}_{c}$, we will assume $\mathbf{R}_{s}=\mathbf{I}$ and $\mathbf{R}_{n}=\sigma_{n}^{2} \mathbf{I}$, to study the asymptotic behavior.

For $\sigma_{n}^{2} \longrightarrow \infty$, both methods, (7) and (12), converge to the same expression, corresponding to the decorrelating matched filter (DMF) solution (up to a scalar factor):

$$
\mathbf{M}_{s} \propto \mathbf{C}^{H} \mathbf{M}_{c} \propto \mathbf{C}^{H} \mathbf{H}_{c}^{H} \mathbf{R}_{n}^{-1} .
$$

On the other hand, for $\sigma_{n}^{2} \longrightarrow 0$, the use of the structured $\mathbf{R}_{c}$, (7) and (11), converges to the symbol-level zero-forcing (ZF) solution:

$$
\mathbf{C}^{H} \mathbf{M}_{c}=\mathbf{M}_{s}=\left(\mathbf{C}^{H} \mathbf{H}_{c}^{H} \mathbf{R}_{n}^{-1} \mathbf{H}_{c} \mathbf{C}\right)^{-1} \mathbf{C}^{H} \mathbf{H}_{c}^{H} \mathbf{R}_{n}^{-1}
$$

whereas the use of the non-structured $\mathbf{R}_{c}$ (12) converges to the chip level zero-forcing solution:

$$
\mathbf{C}^{H} \mathbf{M}_{c}=\mathbf{C}^{H}\left(\mathbf{H}_{c}^{H} \mathbf{R}_{n}^{-1} \mathbf{H}_{c}\right)^{-1} \mathbf{H}_{c}^{H} \mathbf{R}_{n}^{-1} .
$$

Asymptotic expressions (14) and (15) are both unbiased and have an error covariance matrix given by:

$$
\begin{gathered}
\left(\mathbf{C}^{H} \mathbf{H}_{c}^{H} \mathbf{R}_{n}^{-1} \mathbf{H}_{c} \mathbf{C}\right)^{-1} \\
\mathbf{C}^{H}\left(\mathbf{H}_{c}^{H} \mathbf{R}_{n}^{-1} \mathbf{H}_{c}\right)^{-1} \mathbf{C}
\end{gathered}
$$

respectively. It can be shown (see Appendix) that $\mathbf{C}^{H}\left(\mathbf{H}_{c}^{H} \mathbf{R}_{n}^{-1} \mathbf{H}_{c}\right)^{-1} \mathbf{C} \geq\left(\mathbf{C}^{H} \mathbf{H}_{c}^{H} \mathbf{R}_{n}^{-1} \mathbf{H}_{c} \mathbf{C}\right)^{-1}$, where $\mathbf{A} \geq \mathbf{B}$ means that $(\mathbf{A}-\mathbf{B})$ is positive semidefinite. This clearly shows that the utilization of the structured $\mathbf{R}_{c}$ (more information is used) gives a better (or at least equal) performance.

In fact, the Cramer-Rao bound (CRB) for an unbiased estimator is given by:

$$
\mathbf{J}^{-1}=\left(\mathbf{C}^{H} \mathbf{H}_{c}^{H} \mathbf{R}_{n}^{-1} \mathbf{H}_{c} \mathbf{C}\right)^{-1}
$$

and coincides with the error covariance matrix of the block filtering using the structured $\mathbf{R}_{c}$.

\section{Performance eValuation of SPaCe-time MMSE BLOCK FILTERING}

Given a received signal model and an estimated space-time matrix, it is quite simple to estimate its performance in terms of signal-to-noise ratio (SNR). Assuming $E\{s\}=0$ and zeromean noise, the SNR at the output of the block equalizer can be expressed as:

$$
\gamma=\frac{\text { variance of desired signal }}{\text { variance of ISI+MAI+noise }}
$$

With the SNR at the input of the data detector, the average (over users and discrete time) uncoded bit error probability can be estimated as:

$$
\bar{P}_{e}=\frac{1}{K \cdot N} \sum_{k=1}^{K} \sum_{n=1}^{N} \int_{0}^{\infty} P_{b}\left(\gamma_{n}^{(k)}\right) p\left(\gamma_{n}^{(k)}\right) d \gamma_{n}^{(k)}
$$

where $k$ is the user index, $n$ represents the discrete time, $p\left(\gamma_{n}^{(k)}\right)$ is the probability density function of the SNR resulting from the varying channel impulse responses ${ }^{6}$ and $P_{b}(x)$ is the well-known bit error probability for a QPSK signaling over an additive white Gaussian noise (AWGN) channel as a function of the SNR [1]:

$$
P_{b}(\mathrm{SNR})=Q(\sqrt{\mathrm{SNR}})
$$

where $Q(x)=\frac{1}{\sqrt{2 \pi}} \int_{x}^{\infty} e^{-t^{2} / 2} d t[1]$. Note that the bit error probability computed this way is merely an estimate, since the AWGN assumption is only an approximation of the interfering terms.

The estimation of the symbols after the space-time block filtering can be compactly written as:

$$
\hat{\mathbf{s}}=\mathbf{A s}+\mathbf{B n}
$$

${ }^{6}$ Instead of using a theoretical expression for the pdf Monte-Carlo simulations are used to obtain different channel realizations, yielding a semi-analytical performance evaluation method. 


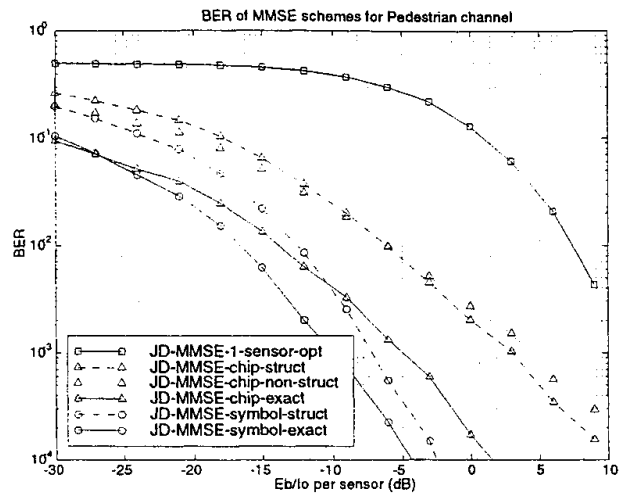

Figure 3: BER of different MMSE configurations for Pedestrian channel propagation model $(\mathrm{v}=3 \mathrm{~km} / \mathrm{h})$ with $A S=8^{\circ}$.

where matrices $\mathbf{A}$ and $\mathbf{B}$ depend on the applied method. The signal estimated for user $k$ at discrete time $n$ can be then decomposed into:

$$
\begin{aligned}
\hat{\mathbf{s}}_{n}^{(k)} & =\underbrace{[\mathbf{A}]_{i, i} \mathbf{s}_{n}^{(k)}}_{\text {desired }}+\underbrace{\left([\mathbf{A}]_{i,:} \mathbf{s}-[\mathbf{A}]_{i, i} \mathbf{s}_{n}^{(k)}\right)}_{\text {ISI }+ \text { MAI }}+\underbrace{[\mathbf{B}]_{i,:} \mathbf{n}}_{\text {noise }} \\
i & =N(k-1)+n
\end{aligned}
$$

where $[\mathbf{X}]_{i, i}$ represents the element in the $i$ th row and $i$ th column of the matrix $\mathbf{X}$ and $[\mathbf{X}]_{i}$, the $i$ th row of $\mathbf{X}$.

From expression (23), it is easy to derive the SNR at the output of the space-time block filter(see (24) on top of next page).

Note that for an ideal knowledge of the channel and noise covariance matrix, perfect estimates of the block filtering matrices can be obtained, leading to the particular SNR expressions given in [11].

\section{SimUlation RESULTS}

Monte-Carlo simulations were carried out assuming the reception of 8 intracell and 6 intercell low-rate users (fixed spreading code with $\mathrm{SF}=16$ ) impinging over an uniform linear array (ULA) of 8 sensors separated half the carrier wavelength (see [14] for downlink single-antenna numerical results using time-varying spreading codes). The direction-of-arrival (DOA) of each source was chosen randomly over the range [$60^{\circ}, 60^{\circ}$ ] (assuming a three-sectorial cell deployment). Imperfect power control (using a $3 \mathrm{~dB}$ step) was considered for all the users with respect to their corresponding base station. All the details of the simulations were chosen according to the 3GPP technical specification for TDD mode of UTRA [3]. The spatial distribution of the sources was modelled using a Laplacian Power Angular Spectrum with a power angular spread (AS) of 8 degrees according to [15]. The temporal dispersion of the channel was generated with both Pedestrian and Vehicular channel propagation models as specified by ETSI [16] with a speed of $3 \mathrm{~km} / \mathrm{h}$ and $120 \mathrm{~km} / \mathrm{h}$ respectively.

According to the scheme depicted in Figure 2, there are just three different cases to compare in UTRA-TDD (the training sequences are defined on a chip-level basis): 1) structured symbol-rate filtering (being identical to structured chip-rate

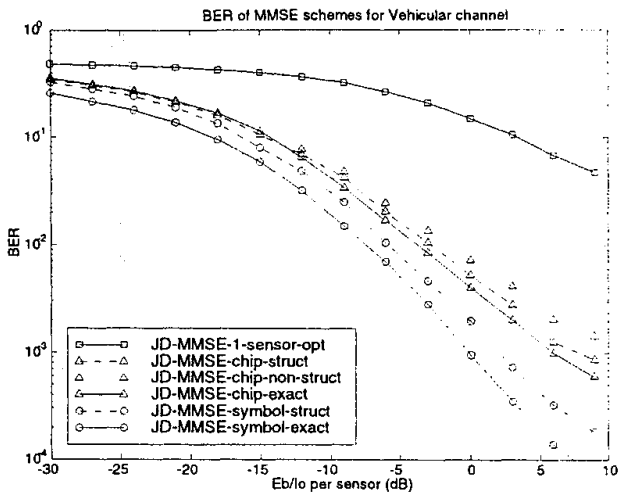

Figure 4: BER of different MMSE configurations for Vehicular channel propagation model $(\mathrm{v}=120 \mathrm{~km} / \mathrm{h})$ with $\mathrm{AS}=8^{\circ}$.

filtering using the structured matrix), 2) non-structured chiprate filtering, and 3) structured chip-rate filtering using the unstructured matrix.

The performance of each methods is given in terms of uncoded bit error rate (BER) as a function of the instantaneous received $E_{b} / I_{0}$ (where $I_{0}$ accounts for all the intercell interfering users) received at one antenna (with $E_{c} / N_{0}=20 \mathrm{~dB}$ ).

It is important to remark that, in a real implementation, it is unaffordable to estimate filtering matrix $\mathbf{M}$ for the whole block (the length of the received vector $\mathbf{x}$ for a block length of 2560 chips and 8 antenna elements is about 20480) and to perform a one-shot block filtering of the received snapshot $\mathbf{x}$. Therefore, it renders necessary to work on a narrow sliding window basis with the consequent performance degradation.

In Figures 3 and 4, the BER of the three aforementioned methods using an estimated channel along with the corresponding to the ideal channel knowledge is depicted for both Pedestrian and Vehicular channel. Remarkably, the BER curves obtained directly from Monte-Carlo simulations or indirectly from the computed SNR using the AWGN approximation were almost identical. As expected from the analytical derivations, for low SNR both symbol-rate and chip-rate filtering methods converge to the DMF, whereas for high SNR, the symbol-rate filtering outperforms the chip-rate filtering (see Figure 3). For the Vehicular model, due to the time-varying nature of the channel coefficients (and the impossibility to estimate it properly), the difference of performance of all the methods is significantly reduced. It is important to remark that the interference-plus-noise covariance matrix has been assumed identical for both the training and the data parts. In practice, taking into account intercell users (interference) and assuming a chip-level training sequence, $\mathbf{R}_{n}$ is temporally white for the training part but structured for the data part (due to the spreading codes), leading to a reduction of performance as can be seen from Figure 5 (synchronization of intercell users is assumed).

\section{CONCLUSIONS}

In the present paper, differences arising from the general MMSE space-time block equalization approach have been stated, depending on whether the training sequence is given at a chip or symbol level, the chip/symbol rate nature of the 


$$
\gamma_{n}^{(k)}=\frac{\left|[\mathbf{A}]_{i, i}\right|^{2} E\left\{\left|\mathbf{s}_{n}^{(k)}\right|^{2}\right\}}{\left[\mathbf{A R}_{d} \mathbf{A}^{H}\right]_{i, i}+\left|[\mathbf{A}]_{i, i}\right|^{2} E\left\{\left|\mathbf{s}_{n}^{(k)}\right|^{2}\right\}-2 \operatorname{Re}\left\{\left[\mathbf{A R}_{d}\right]_{i, i}[\mathbf{A}]_{i, i}^{*}\right\}+\left[\mathbf{B R}_{n} \mathbf{B}^{H}\right]_{i, i}}
$$

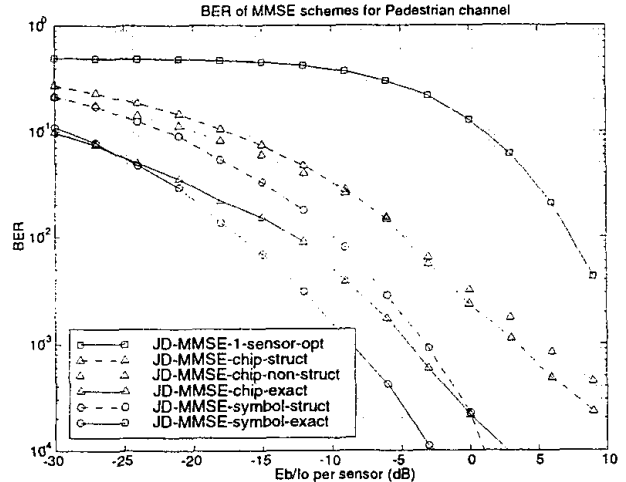

Figure 5: BER of different MMSE configurations for Pedestrian channel propagation model $(\mathrm{v}=120 \mathrm{~km} / \mathrm{h})$ with $A S=8^{\circ}$, assuming a realistic model for $\mathbf{R}_{n}$.

filtering process and the non-structured vs. structured computation of the block filtering matrix. According to these alternatives, different variants of the MMSE method naturally arise. To be more specific, only three of these schemes apply to TDD mode of UTRA. They have been analytically compared and extensively tested via Monte-Carlo simulations.

The main conclusion to be drawn upon the results of this paper is that MMSE symbol-rate filtering clearly outperforms chip-rate alternative schemes.

\section{APPENDIX}

Proof that $\mathbf{C}_{1}^{H}\left(\mathbf{H}_{c}^{H} \mathbf{R}_{n}^{-1} \mathbf{H}_{c}\right)^{-1} \mathbf{C}_{1} \geq\left(\mathbf{C}_{1}^{H} \mathbf{H}_{c}^{H} \mathbf{R}_{n}^{-1} \mathbf{H}_{c} \mathbf{C}_{1}\right)^{-1}$.

Assuming that $\mathbf{H}_{c}$ is full column rank (which is true for linearly independent channels for each user at least at one receiving antenna), then $\mathbf{H}_{c}^{H} \mathbf{R}_{n}^{-1} \mathbf{H}_{c}$ is positive definite. Since $\mathbf{C}_{1}$ is a "tall" matrix with orthonormal columns $\left(\mathbf{C}_{1}^{H} \mathbf{C}_{1}=\mathbf{I}\right)$, we can complete the set of columns to get a unitary matrix $\mathbf{C}=\left[\begin{array}{ll}\mathbf{C}_{1} & \mathbf{C}_{2}\end{array}\right]$ so that $\mathbf{C}^{H} \mathbf{C}=\mathbf{C C}^{H}=\mathbf{I}$. Using the decomposition $\mathbf{H}_{c}^{H} \mathbf{R}_{n}^{-1} \mathbf{H}_{c}=\mathbf{U D U} \mathbf{U}^{H}$, we can define the matrix $M$ as:

$$
\mathbf{M}=\mathbf{C}^{H} \mathbf{H}_{c}^{H} \mathbf{R}_{n}^{-1} \mathbf{H}_{c} \mathbf{C}=\tilde{\mathbf{C}}^{H} \mathbf{D} \tilde{\mathbf{C}}
$$

where $\overline{\mathbf{C}}=\mathbf{U}^{H} \mathbf{C}$.

Since $\mathbf{H}_{c}^{H} \mathbf{R}_{n}^{-1} \mathbf{H}_{c}$ is definite positive, so is $\mathbf{M}$ and we have the following inequality [17, p.474, Theorem 7.7.8]:

$$
\left(\mathbf{M}^{-1}\right)(S) \geq(\mathbf{M}(S))^{-1}
$$

where $\mathbf{M}(S)$ is the principal submatrix of $\mathbf{M}$ determined by deletion of the rows and columns indicated by the index set $S$. Using the fact that $\mathbf{M}^{-1}=\tilde{\mathbf{C}}^{H} \mathbf{D}^{-1} \overline{\mathbf{C}}$, it follows that $\tilde{\mathbf{C}}_{1}^{H} \mathbf{D}^{-1} \overline{\mathbf{C}}_{1} \geq\left(\tilde{\mathbf{C}}_{1}^{H} \mathbf{D} \tilde{\mathbf{C}}_{1}\right)^{-1}$ and, therefore, $\mathbf{C}_{1}^{H}\left(\mathbf{H}_{c}^{H} \mathbf{R}_{n}^{-1} \mathbf{H}_{c}\right)^{-1} \mathbf{C}_{1} \geq\left(\mathbf{C}_{1}^{H} \mathbf{H}_{c}^{H} \mathbf{R}_{n}^{-1} \mathbf{H}_{c} \mathbf{C}_{1}\right)^{-1}$. QED.

\section{REFERENCES}

[1] J. G. Proakis, Digital Communicutions. McGraw-Hill, 3rd ed., 1995.

[2] R. L. Peterson, R. E. Ziemer, and D. E. Borth, Introduction to Spread Spectrum Communications. Prentice Hall, 1995.

[3] 3GPP, "Physical channels and mapping of transport channels unto physical channels (TDD)," TS 25.221 (v3.0.0), TSC, RAN, WG1, http://www.3gpp.ory, Oct. 1999.

[4] S. Mushavi, "Multi-user detection for DS-CDMA communications," IEEE Comm. Magazine: Topics in Personal Communications, pp. 124-136, Oct. 1996.

[5] S. Verdú, "Minimum probability of error for asynchronous gaussian multiple-access channels," IEEE Trans. on Informution Theory, vol. 32, pp. 85-96, Jan. 1986.

[6] R. Lupas and S. Verdú, "Linear multiuser detectors for synchronous code-division multiple access channels," IEEE Trans. on Information Theory, vol. 35, pp. 123-136, Jan. 1989

[7] Y. Cho and J. H. Lee, "Analysis of an adaptive SIC for nearfar resistant DS-CDMA," IEEE Trans. on Communications, vol. 46, pp. 1429-1432, Nov. 1998.

[8] P. Patel and J. Holtzman, "Analysis of a simple successive interference cancellation scheme in a DS/CDMA system," IEEE Journal on Select. Areas in Communications, vol. 12, pp. 796807 , June 1994 .

[9] A. Klein and P. W. Baier, "Linear unbiased data estimation in mobile radio systems applying CDMA," IEEE Journal on $S E$ lected Areas in Communications, vol. 11, pp. 1058-1066, Sept. 1993.

[10] A. Klein, G. K. Kaleh, and P. W. Baier, "Zero forcing and minimum mean-square-error equalization for multiuser detection in code-division multiple-access channels," IEEE Trans. on Velicular Technology, vol. 45, pp. 276-287, May 1996

[11] P. Jung and J. Blanz, "Joint detection with coherent receiver antenna diversity in CDMA mobile radio systems," IEEE Truns. on Vehicular Technology, vol. 44, pp. 76-88, Feb. 1995.

[12] I. Ghauri and D. T. Slock, "Linear receivers for the DS-CDMA downlink exploiting orthogonality of spreading sequences," in Conf. Rec, 32nd Asilomar Conf. Signals, Systems and Computers, (Pacific Grove, CA), pp. 650-654, Nov. 1998.

[13] T. P. Krauss, M. D. Zoltowski, and G. Leus, "Simple MMSE equalizers for CDMA downlink to restore chip sequence: comparison to zero-forcing and Rake," in Proc. IEEE-ICASSP 2000, vol. V, (Istanbul (Turkey)), pp. 2865-2868, June 5-9, 2000 .

[14] K. Hooli, M. Juntti, and M. Latva-aho, "Inter-path interference suppression in wcdma systems with low spreading factors," in IEEE Velicular Tech. Conf., vol. VTC-1, (Amsterdam, The Netherlands), pp. 421-425, Sept. 19-22, 1999.

[15] K. I. Pedersen, P. E. Mogensen, and B. H. Fleury, "Spatial channel characteristics in outdoor environments and their impact on BS antenna system performance," in Proc. VTC'98 (Ottawa, Canada), pp. 719-723, May 1998.

[16] ETSI, "Selection Procedures for the Choice of Radio Transmission Technologies of the UMTS (UMTS 30.03)," Tech. Rep. TR101112, v3.2.0,ETSI, 1998

[17] R. A. Horn and C. R. Johnson, Mutrix Analysis. New York: NY: Cambridge University Press, 1985. 